\title{
Recorded Work Meetings and Algorithmic Tools: Anticipated Boundary Turbulence
}

\author{
Peter W. Cardon \\ University of Southern California \\ cardon@marshall.usc.edu
}

\author{
Haibing Ma \\ DeepBlue Technology \\ University of Southern California \\ mahb@deepblueai.com
}

\author{
Carolin Fleischmann \\ Ansbach University \\ of Applied Sciences \\ carolin.fleischmann@hs-ansbach.de
}

\begin{abstract}
Meeting recordings and algorithmic tools that process and evaluate recorded meeting data may provide many new opportunities for employees, teams, and organizations. Yet, the use of this data raises important consent, data use, and privacy issues. The purpose of this research is to identify key tensions that should be addressed in organizational policymaking about data use from recorded work meetings. Based on interviews with 50 professionals in the United States, China, and Germany, we identify the following five key tensions (anticipated boundary turbulence) that should be addressed in a social contract approach to organizational policymaking for data use of recorded work meetings: disruption versus help in relationships, privacy versus transparency, employee control versus management control, learning versus evaluation, and trust in AI versus trust in people.
\end{abstract}

\section{Introduction}

While online meetings have been an increasingly common approach to business communication for well over a decade, the COVID-19 pandemic has dramatically accelerated the use of online meetings. One measure of the rapid growth of online meetings is from the vendor Zoom. From January to March 2020, daily active users on Zoom rose from 10 million to 200 million [1]. A wide array of AI tools is used to provide live captioning and translation, create transcripts, and evaluate data from recorded meetings. Various algorithmic tools can evaluate this data to measure employee engagement, communication performance, team dynamics, and other aspect of interpersonal interactions. These algorithmic tools not only diagnose, they often provide recommendations to improve interpersonal communication, team performance, organizational culture, and to assist in hiring and promotion decisions [2] [3] [4] [5] [6] [7] [8].

Employers relying on the data from employees' digital footprints to provide insights and inform decisions is not new [9]. Yet, the data from recorded meetings is distinct from data in traditional digital footprints. First, in-person conversations have rarely if ever been recorded in most workplaces. Generally, employees have held an expectation that these conversations are private and tend to disclose much more in these conversations than they do in email, chat, and other written forms of communication, which tend to be more planned and filtered [10] [11]. Second, it potentially captures a much larger portion of employee communication. In the past, just small parts of our interactions were recorded. Typically, what left a record in our workplace interactions were simply in the forms of text in emails and other written communications. Now, extended, in-depth conversations can be recorded, stored, and shared widely. Third, and perhaps most importantly, AI algorithmic tools can evaluate verbal tone, nonverbal expressions, and conversation transcripts to make judgments and recommendations.

Using algorithmic tools to evaluate recorded meeting data may provide many new opportunities for employees, teams, and organizations. Yet, the use of this data raises important consent, data use, and privacy issues. The purpose of our research was to identify key tensions that should be addressed in organizational policymaking about data use from recorded work meetings. We identify these tensions through interviews with American, Chinese, and German professionals and suggest a social contracts approach to addressing these tensions.

Specifically, five key tensions emerged from these interviews: disruption versus help in relationships, privacy versus transparency, employee control versus management control, learning versus evaluation, and trust in AI versus trust in people. 
Our results contribute to research on the ethical use of virtual meeting recordings and their algorithmic evaluation as called for by Seeber et al. (2019) [12]. For the field of ethics and technology, we contribute a crosscultural view on a fairly new and quickly expanding technology: meeting recordings and their analysis with algorithmic tools. For practice, our findings may guide organizational policymaking and raise awareness of emerging boundary turbulences.

\section{Literature Review}

Norms around data use and privacy related to recorded work meetings are underdeveloped. Further, the development of algorithmic tools to evaluate this data are just emerging and will likely present many unforeseen scenarios. In this literature review, we broadly lay the foundation for our study with discussions of workplace privacy, algorithmic tools used for employee data, and a social contracts approach to developing privacy and data use policies.

\subsection{Workplace privacy}

Workplace privacy has been studied extensively over many decades. Among the foremost scholars in workplace privacy are Stone \& Stone-Romero [13] [14] [15], who define workplace privacy as "a state or condition in which the individual has the capacity to (a) control the release and possible subsequent dissemination of information about him or herself, (b) regulate both the amount and nature of social interaction, (c) exclude or isolate him or herself from unwanted (auditory, visual, etc.) stimuli in an environment, and, as a consequence, can (d) behave autonomously (i.e., free from the control of others)" [13]. Most research about workplace settings focuses on invasions of privacy (e.g., interview process and employee selection, electronic monitoring). Our focus is primarily on information privacy, which involves "control over the acquisition, storage, use, dissemination, and dispersal of employees' data. That is, it concerns control over the information that could be made available to others" [2]. Technological developments have been the primary driver of renewed interest in workplace privacy in recent years [2] [16]. Advances in technology create privacy dilemmas much faster than people can foresee and resolve them, particularly with AI algorithms [17] [18].

Professionals tend to develop a privacy calculus. They make cost-benefit judgments about whether they should disclose information. Further, professionals tend to negotiate, implicitly or explicitly privacy rules. When one party breaks those rules, the outcome is boundary turbulence [2] [19]. Research shows that the privacy calculus varies across cultures. For example, one study showed members in high uncertainty avoidance cultures were more concerned about privacy. This study included samples from Germany (high uncertainty avoidance), the United States (moderate uncertainty avoidance), and China (low uncertainty avoidance) [20]. Another study compared the social media attitudes of Americans (more individualist and higher in uncertainty avoidance) and Chinese (more collectivist and lower in uncertainty avoidance), suggesting individualism is associated with higher self-disclosure, and collectivism is associated with more reliance on group norms [21]. Other scholarly works suggests Americans are more concerned about information privacy from the government, whereas Europeans are more concerned about information privacy from corporations [22]. Often, privacy norms are reflected in regulation, with the European Union likely the most stringent regulator. Perhaps the most influential regulation is the GDPR in the EU [2]. Within American organizations, existing digital footprints in companies are generally not considered private, even though the rationale for monitoring employee digital footprints from email and other activities is often not ethically justified [23]. Yet, little cross-cultural work focuses on the application of new and emerging forms of tools and their affordances (i.e., algorithmic evaluation of recorded meeting data) within organizations.

\subsection{Algorithmic evaluation of employee data}

A variety of algorithmic approaches are being applied to evaluating employees' and prospective employees' data from online meetings and team messaging platforms. Using facial recognition, voiceto-text, natural language processing, sentiment analysis, machine learning, and other AI technologies, these tools often assess and evaluate data from online meetings and online chat to measure communication performance, communication breakdowns, team effectiveness, team dynamics, employee engagement, employee sentiment (e.g., happiness, excitement, depression), and employee productivity. These algorithmic tools can be diagnostic in nature and may also make recommendations. Often, this data can be used in predictions of organizational commitment and organizational tenure. They can be used on individual, interpersonal, team, and organization-wide levels [2] [3] [4] [5] [6] [7] [8].

While many algorithmic tools can be applied to emerging forms of employee data, such as recorded meeting data, the purposes and goals of the tools emerge from different paradigms. For example, people analytics tools are typically developed with a human relations perspective, with focus on employee satisfaction, employee growth and development, and employee 
career opportunities [24]. On the other hand, productivity and electronic surveillance tools focus more so on ensuring employees remain on task and avoiding risk to organizations [2] [25].

Gal and colleagues' work has focused on the three ethical consequences - opacity, datafication of the workplace, and nudging — of these sophisticated people analytics tools. They suggest the proliferation of people analytics tools, including those relying on recorded meeting data, as "the era of algorithmic management." [24] Other scholars suggest that this era of algorithmic management gives managers control at the expense of employees through the mechanisms of restricting and recommending, recording and rating, and replacing and rewarding. [26]

\subsection{A social contracts approach to emerging norms related to recorded meeting data}

The role of AI algorithms in the workplace raises challenging ethical issues. In the context of collaborating with autonomous agents, one group of global scholars has identified the many possible unintended consequences of algorithms. They advocate for additional research and specifically call for the continued development of ethical frameworks through policymaking and advisory organizations such as the OpenAI initiative and GDPR. They suggest that rules for ethical and unbiased algorithms should address the following issues: "who gets to decide, (2) who is accountable, (3) how can tech-agents be audited, and (4) who takes responsibility so that such agents are beneficial for humanity." [12]

Recorded work meetings will dramatically increase the amount of employee communication that is potentially available to organizations. It will raise data use, privacy, and consent issues that are profoundly different than those of email communications and other traditional forms of business communication. Further, we suggest that in addition to societal-level guidelines for algorithmic tools, organizations should engage their employees in developing guidelines for appropriate use. Given that corporate informed consent processes are often intentionally obfuscating [27] and given the growing complexity of data use from recorded work meetings, we ground our work in a social contracts view of ethics. This approach ensures that all stakeholders, including employees, should have input about policies related to data use [28].

Recent scholarly work by Martin suggests digital information should be governed by a social contract approach [29] [30]. Firms should be responsible for engaging their communities in privacy norm generation. Martin provides an overview of three schools of thought about privacy expectations: the access view, the control view, and the social contract view. In the access view, people give up their right to privacy when they voluntarily share information (e.g., posting online). In the control view, people give up their right to privacy when they agree to give information to another party (e.g., signing notice and choice statements). In the social contract view, privacy norms are the "unstated agreements that individuals and groups make in contexts, communities, and relationships." It is the obligation of organizations to develop norms of privacy with its employees and stakeholders. In the social contract view, privacy is defined as "negotiated information norms within a particular community or situation." The social contract view is fundamentally based in the notion that privacy is contextually- and relationship-dependent. Privacy rules are negotiated within particular communities as microsocial and macrosocial contracts [29].

\section{Methodology}

The purpose of our research is to identify key tensions that arise from recording meetings and that would inform a social contract approach to organizational policy making. Specifically, our research is informed by the following research question: How do professionals think the data from recorded meetings should and should not be used? In what ways should algorithmic tools be applied or not applied to recorded meeting data? By speaking to professionals across organizations and cultures, our goal was to identify key issues that should be addressed by stakeholders in a social contract approach to organizational policymaking.

Because our research is exploratory, we chose to conduct interviews so we could gain in-depth views perspectives with a lot of follow-up questions. We chose purposive sampling and specifically sought professionals who are early adopters of technology and who had held management roles [31]. This allows them to provide a forward-looking perspective on key issues from the vantage points of managers as well as employees. We chose to interview professionals in three countries: the United States, China, and Germany. These represent the largest economies in North America, Asia, and Europe. These country selections match the expertise of our research team, which is comprised of four multilingual scholars. The team includes a native English speaker from the United States, a native German speaker from Germany, and a native Chinese speaker who is a Chinese national. All members of the research team are fluent English speakers, and the team also includes second-language German and Chinese speakers. 
Altogether, we interviewed 50 professionals during May and June 2020. Participants had an average of 15 years of work experience and a median of 13 years of work experience. Respondents came from a range of industries, including technology, financial services, professional services, medical, retail, education, and aerospace and defense. The first batch of interviews were conducted among 24 American professionals. Next, 15 Chinese professionals and 11 German professionals were interviewed. Our team identified the point of data saturation at about 15 interviews among the American sample and at about 10 interviews among the Chinese and German samples [32].

In preparation for interviews, participants viewed a five-minute video about the research goals of the project. Participants saw several use cases of contemporary meeting tools that rely on various AI technologies and algorithms to evaluate communication performance. These examples included virtual meeting assistants, automated notes tools, and team dynamics tools. Semi-structured interviews lasted from 30 to 60 minutes. Respondents were asked questions such as the following: What types of meetings should be recorded? What types of meetings shouldn't be recorded? Who do you think recorded meetings should be shared with? Who shouldn't they be shared with? Should there be rules about consent to be recorded? Should there be rules about the data is used? What do you think about AI tools to evaluate recorded meetings? What are some types of AI tools that would be helpful? Not helpful? In what ways is it appropriate to use AI tools to evaluate recorded meeting data? All interviews were recorded and transcribed. The Chinese interviews were translated into English. All but one of the German interviews were conducted in English. The one interview in German was also translated into English.

We started by independently reading interview transcripts. Then, we engaged in several rounds of independent coding that helped us identify subcodes, codes, and ultimately themes [33]. All transcripts were coded by at least two members of the research team. Our independent coding was aligned in nearly all cases. In rare cases when we coded passages differently, we discussed as a team what the codes and relationships should be. Most of our themes involved tensions in which participants adopted widely contrasting views.

\section{Findings}

In our preliminary analysis, we identified two contextual factors that help explain participants' views of recorded meetings: pre-existing attitudes toward technology and national culture. We identified five key tensions in how algorithmic tools could be applied to recorded meeting data: disruption versus help in relationships, privacy versus transparency, employee control versus management control, learning versus evaluation, and trust in AI versus trust in people. These tensions are what we anticipate will likely create boundary turbulence as these algorithmic tools become more commonplace.

\subsection{Preexisting attitudes toward technology}

The existing attitudes toward AI and workplace productivity tools significantly informs how professionals view new technologies, including AI tools for recorded meetings. Participants in this study varied significantly in terms of prior experience with recorded meetings, personal preferences for privacy, and the perceived reliability in emerging AI analytical tools.

Generally, American participants had the most experience with recorded meetings. They ranged from periodically recording meetings to recording all meetings, with an average of roughly 25 to 30 percent of meetings being recorded. Many American participants had experimented with transcript technologies, virtual meeting assistants, and AIpowered presentation coaching technologies. Chinese and German participants recorded far fewer meetings and were less familiar with emerging AI tools for recorded meetings.

Participants often held divergent views about how reliable and useful various tools could be in evaluating communication performance. Referring to a tool that would evaluate conversational patterns, a German professional explained, "I'd say there are no objective metrics in this case. . I mean, even if the AI software says that you have talked $60 \%$ of the interview, then the question is, was that good or not? ... And I'm not sure if the software can really judge what's been happening. So, I think it's always some kind of subjective based thing. . . The target is 'is the customer satisfied?' For example, that's our main target. It should never be measured by such a software or maybe it can, in the future, but right now I don't really see it." A Chinese professional commented about the quality of the software, "Many current tools make errors when transcribing a recording from voice into text. The current accuracy rate is barely acceptable. If you participate in the meeting personally, it is ok. If you are not involved in this meeting, reading the text transcription will be kind of difficult. Therefore, the accuracy of the transcription is currently a bottleneck."

Yet, many participants, particularly American respondents, expressed confidence in the usefulness of the tools and expressed eagerness to use them if the benefits were clear. This optimism is reflected in many of the comments throughout the remainder of the article. 


\subsection{National culture and conditions}

Throughout the Findings section, we report various tensions. Often, we report how Americans, Germans, and Chinese varied on these tensions. Generally, these variations appeared to be due to several factors. First, there is a significant difference in how data privacy is regulated. Germany follows the General Data Protection Regulation (GDPR) guidelines created for EU countries. This is the strictest set of data privacy guidelines in the world. Nearly all participants in Germany mentioned the GDPR in their interviews. Participants in China and the U.S. were less likely to know what the relevant laws or regulation were, often suggesting they needed to be developed. Second, norms for recorded meetings vary. For example, many German and Chinese participants mentioned they kept their cameras off in online meetings, whereas all American participants explained they kept their cameras on. These variations significantly influence the types of recorded meeting data that can be collected and analyzed with AI tools. Third, there are significant differences in the level of technology adoption for online meetings and related tools. AI tools for recorded meetings tend to be more widely adopted in the U.S., and development of these tools has been ongoing for many years by Silicon Valley firms. Finally, underlying cultural differences related to norms and values, such as power distance and uncertainty avoidance, have some impact [34]. For example, Chinese participants, considered higher in power distance, were much more likely to defer to authorities in policymaking. Germans, considered much higher in uncertainty avoidance, were much more likely to appeal to laws and regulations.

\subsection{Disruption versus help in relationships}

Participants varied significantly on the basic premise of whether AI tools would disrupt or help professional relationships. Many viewed AI tools applied to recorded meeting data as disruptive because they would lead to inauthentic behavior and loss of psychological safety. On the other hand, many viewed AI tools as potentially building more honest and inclusive relationships.

One of the most pervasive reactions among participants was the fact that recording meetings make people less candid and open, especially if they know algorithms will be used to evaluate the meetings. A German professional explained, "I mean, consulting is a lot about presenting and selling yourself right. So, this can help maybe to get a picture about your employees, but I don't think that it's the whole picture because people, from my point of view, I think that people behave differently in front of a camera than if we are face to face in the meeting room." Some professionals thought filtering one's behavior wasn't necessarily a bad thing. Rather, they suggest it leads to more professional behavior. For example, a German professional said, "I think that [being recorded] can be a good thing if you are thinking more about what you are telling your clients or your colleagues."

A common issue raised by participants was that the diminished role of people could lead to less psychological safety. An American professional explained, "It goes back to that psychological safety. . . If I knew I was being recorded, I would definitely be more reserved about what I said." A German professional said, "Actually, it will be scary. Well, maybe for me in the management position, it would be interesting, but I would not want it because I think it would also kind of destroy our corporate culture, which is also based on trust. It's very much that it's very much control focus there."

Yet, others saw promise in creating more productive and psychological safe environments. One German professional explained, "In the far future, AI would help you to identify conflict in the very early stages. Then, this could be used to prevent escalation and down spiraling worst case scenarios. So, if you could say, hey, these two people will have a meltdown in about four weeks and say that because tone of voice, staring off the eyes, and I don't know." Similarly, many participants saw promise in tools that improved meetings and ultimately team dynamics. An American professional explained, "How effective was the meeting? And I think with there's transcripts, there will be a algorithms to determine whether a meeting was effective, whether it was productive, whether action items were done, whether there were some, you know, what was the intention of the meeting in the first place, and was the result. So, by having this and then having the ability to scan through with AI, I think we will get to a point where we will be able to ask those types of questions from transcripts."

American participants were particularly attracted to the idea that AI could help identify and encourage those who don't speak up. One participant explained, "If you know some part of the population is holding back or, you know, they are not speaking enough, that kind of analysis of body language - that's number one. And number two, we can go back and check what kind of content people share and how that is impacting a product decision and you know whether everybody speaking up in a meeting is providing a better product decision."

Many participants saw positive value to improve interpersonal skills and foster team building. A German professional explained, "I would love to use it in mainly in coaching my employees. . or it could be actually used actively for moderating or creating better meetings 
in the future." Some participants even emphasized that AI tools might provide feedback in non-threatening ways. An American participants explained, "Without a program manager or note taker in these meetings, one person would go off and speak $90 \%$ of the time, and then not get feedback from other team members with their thoughts that I felt like there were a lot of inefficiencies there. So to have an unbiased technology telling you these are how your meetings are run, people may not take it as offensively and they may actually see it as like, 'Oh, I could actually learn from this feedback.' And not take it personally. And I think that probably is an interesting value proposition."

\subsection{Privacy versus transparency}

Participants varied significantly in terms of how invasive it is to record and analyze meetings with AI tools. Many thought this use of AI crossed a boundary of privacy that was unacceptable. Yet, many others were willing to give up privacy for the sake of transparency as long as there were advantages for employees.

Among professionals who were particularly sensitive to the loss of privacy, they often were concerned about the feeling of being surveilled, even in their thoughts. An American professional explained "I think there needs to be some boundaries where you have freedom to say and do things that are not recorded. . . it's going to create this weird culture of big brother's always watching and then, you know, we sort of get that guarded. It doesn't foster authenticity." A Chinese participant explained, "Personally, I really don't like this kind of thing [evaluation tools]. Because they make people lose even the one minute of privacy they had left."

On the other hand, some respondents, particularly among American participants, thought the benefits of transparency and other advantages far exceeded the drawbacks of less privacy. For example, an American professional suggested the following: "I think that that transparency has to be part of the culture of the company and has to be explicit. When someone onboards, a company can show that this [AI tools for recorded meeting data] is something that's being used to improve the company, develops benefits for them, puts teams together. It provides opportunities for them. If they can show the benefit of using AI for their employees, by all means, as long as they know that it's happening. . . I think it's fine. I think it's something that as long as you're transparent about what you're doing." Another American mentioned, "I think if I knew how the data was being used. I think I would be okay with it. If it could improve my work experience."

\subsection{Employee control versus management control}

When it comes to control over recorded meeting data, participants often distinguished between power held by employees versus that held by managers. Overwhelmingly, participants stated there should be opt-in mechanisms to give employees some control. In practice, these opt-in mechanisms generally involve direct requests to record meetings and pop-up consent boxes. Many professionals explained they asked for permission to record at least a day in advance. Yet, much more variation existed about whether employees could control how data was shared and used after a meeting was recorded. Similarly, some participants wanted the right to control who could see their data. An American professional commented, "I would definitely want to have the option to control who can have access to that information. Like it's my choice if I would like it to keep it to myself. But then if I want, I can share it with anyone I want. So, it should be like more driven by me then." Fewer participants mentioned the right to opt out. An American participant mentioned, "So if I'm in a meeting and they're saying we're recording this meeting, I should be able to hit up a button that says I'm opting out on this one. I want to be able to openly, freely share how I feel. And I don't want somebody analyzing it, and it should be able to strip my analysis out."

Many participants stated there must be clear guidelines and frameworks for how recorded meeting data should be used within organizations. For example, a Chinese respondent explained, "I would definitely communicate with employees and propose written rules. Then I will apply these tools. I will tell them when I will use this algorithm and when I will not use it. I'll do this under a framework." An American professional stated, "It's going to have to be real clear in the guidelines: How will we use the information? What information is being gathered and then how can company ensure that it's being used properly? How can we ensure some employees not going to go rogue and misuse it? And just cast the vision for how it will improve their [employees'] experience, their working experience and how to improve the company and set the company apart." German and Chinese participants were more likely to focus on legal frameworks.

Similarly, some participants expressed the importance of anonymizing data. An American participant said, "At the organizational level, I think you should be allowed to use it [the recorded meeting data] as long as it's anonymous, whether it's, you know, AI specifically or not. Redact names or references that like you know the person, the blue shirt, what have you."

Yet, there was not necessarily consensus on the degree to which employees should be involved. 
Americans varied widely, with some subscribing more so to the control view and some subscribing to the social contract view. Others suggested heavy involvement by employees in the form of task forces and committees. The composition in these working groups that cross functions and layers within organizations. Some proposed ethicists should be involved. Many employees suggested that the guidelines would need to be updated frequently. Germans and Chinese were much more likely to suggest that legislative, regulatory, and worker rights' groups should manage the process.

\subsection{Learning versus evaluation}

Most participants clearly felt more comfortable with AI tools that were restricted to learning, often drawing a line with any form of evaluation, performance review, or potential negative impacts. In many cases, they clearly considered evaluation a breach of trust and psychological safety. A German professional commented, "I can never be myself in the end. . . You are never doing your best work when you afraid of somebody watching you, or of punishment or getting a bad mark. . . I don't really see a big advantage of any of these functionalities - [it's not] creating psychological safety ... We have a leadership culture. For example, at the call center . . . everybody is inherently trustworthy and everybody wants to do a good job." A Chinese participant commented about the distinction between learning and evaluation in this way: "Personalized data is a double-edged sword. If it is used for management, the person being managed will be more uncomfortable. If it is used to help you, it can help you progress. If this has something to do with employees' assessment and performance, or affects their future room for improvement. For example, after evaluation, if their position is not suitable, they will have concerns."

Another American professional emphasized that to gain buy-in from employees for AI tools, it was necessary to emphasize learning rather than evaluation. "Let's say you want to increase engagement and or you want to increase people's communication skills and they are talking to customers. . . And tying it again, back to the goals that they have for the organization as well as for those employees. It could also come up as an individual growth plan. For everyone you know you can also use it as something that would help employees improve themselves and sell it like that, but it just depends on whether employees are interested in it or not."

Yet, participants sometimes mentioned the value of using AI tools for evaluation. As a German professional said, 'People analytics don't replace leader empathy and people behave differently when on camera, but generally it may be helpful for leaders to have access to that data, particularly now that everyone is working remotely and you see less what your people are doing. Employees think it can add to them being fairly evaluated."

\subsection{Trust in AI versus trust in people}

Many participants suggested that people should make decisions, with minimal to no involvement from AI tools. Other participants, however, were optimistic that AI tools could provide trustworthy input in decision-making. At the heart of these differing perspectives tended to be contrasting views about whether people are more biased or whether AI is more biased.

In line with current research [12], many expressed skepticism that algorithms would root out bias. As a result, many participants suggested, at best, these tools should only provide partial input to decision making. For example, a Chinese participant said, "As a manager. I think this tool is useful. But it can only be used as a supplementary tool or as an auxiliary tool. You cannot rely on them completely. You cannot put it in a dominant position. It can only assist in certain aspects of analysis. But you cannot use it to judge and measure whether employees are working hard. Because every employee works in different ways. It cannot be said that this person who is always in silence, does not like be expressive, does not like to show off, and then their evaluation is not good, and they will be replaced. It's unfair. It cannot be used as such a tool."

On the other hand, many participants were intrigued by the notion that AI algorithms could potentially remove or diminish bias in many ways. A German professional said, "I trust data much more than politics and the HR people, to be honest. If something like this would be there for the last two years during our leadership development program monitoring me and the other three candidates that were in the same role, who now have the same position and same salary. Um, I think that the AI would have positioned me somewhere else."

An American professional pointed out the value of these tools to eliminate the bias that occurs when just a few leaders control most decisions. "There's a lot of ... biases. There is a cluster of leaders who just validate their opinions, and the opinions of newcomers. The opinions of new leaders . . . don't always get heard and there's like four or eight people in the organization that make all the decisions. They talk among each other. They've been working together for 10 years. The other ones who kind of escalate things to the highest leadership and decision is being made by the opinion of 
one or two of the entire population. . . If this kind of AI software can tell us, like what amount of the ideas and brainstormed opinions are actually being embraced."

\section{Discussion}

Organizations are increasingly able to track and record their employees' communications. With the proliferation of online meetings, many of which are recorded, many organizations - or at least managersnow have access to extended and in-depth conversations of their employees. Many emerging AI algorithmic tools can evaluate the recorded meeting data and make a variety of recommendations. This raises many ethical issues related to privacy and consent.

We suggest a social contract approach within organizations to develop ethical guidelines related to recorded meeting data. This research highlights that tensions exist about how recorded meeting data should be used within organizations. These tensions reflect the likely outcome of boundary turbulence as algorithmic tools applied to recorded meeting data become more commonplace. As these tools become more frequently used, it's likely many new and unintended outcomes will occur [12], thus necessitating an ongoing conversation around these issues. Without a robust social contract approach that involves many stakeholders, it is likely that employees will believe their privacy expectations have been violated. Interestingly, there is even tension regarding whether the social contract approach should be put into place. Many participants, particularly Americans, effectively agree with the control and access views of privacy. Still, most participants favored a social contact approach that grants employees more input in developing consensus on these issues.

This research provides key issues which organizations should raise in conversations with their employees about their policies related to recorded meeting data and how algorithmic tools should and should not be applied to this data. In research and application, these issues might be evaluated in terms of Kellogg and colleagues' work on algorithmic management, which suggests that control is exerted through the mechanisms restricting and recommending, recording and rating, and replacing and rewarding [26]. For example, many participants talked about the role of algorithmic tools that evaluate team dynamics based on recorded meeting data. This specific context should be exhaustively explored as far as how these algorithmic tools might restrict information and team behavior, recommend various behaviors the team might not take on its own, record and track team behavior, rate team behavior in ways not done in the past, potentially replace team members who are not good fits, and reward team members who are deemed integral to higher team performance.

Our findings demonstrate that professionals are sensitive to "algorithmic management" [24]. Most professionals in this study were concerned about the implementation of these tools in ways that took control from employees and gave it to management. Further, many felt the tools should be primarily be used for learning rather than evaluation. As organizations develop policies surrounding these tools-ideally in a social contracts approach-they should explore how to avoid opacity, datafication, and nudging. Similarly, they should aim for the people analytics approach from a human relations framework rather than a productivity and surveillance framework [24].

The cross-cultural approach to this project is particularly relevant for several reasons. Business professionals increasingly rely on online meetings for their global business communication, and the related AI tools are particularly helpful for captioning and translation. Second, a social contract approach for global companies requires navigating cultural differences among their employees. Also, the AI tools used to evaluate recorded meeting data typically emerge from particular cultures (e.g., Silicon Valley in the United States). Thus, it is crucial to understanding the worldviews and values of these developers. Overall, we recommend further exploration of how cultural dimensions, such as power distance and uncertainty avoidance [34], influence pre-existing attitudes toward these new tools as well as the experiences of professionals as they put them into practice.

Our preliminary analysis suggests that American professionals tend to demonstrate more optimism for these tools, and German and Chinese professionals tend to hold more concerns about privacy. Interestingly, Chinese tended to be more concerned about privacy violations by their companies than by their government, and the opposite appeared to be true for Americans and Germans. Some of these findings aligned with our existing expectations based on national norms and values. Yet, part of these differences may be explained by exposure to these AI tools. These tools tend to be more developed and implemented in American workplace settings, whereas European data regulation (e.g. GDPR) and German workplace norms and practices are restricting the adoption of such algorithmic evaluation tools.

This research has several limitations. First, it lacks representativeness. The value of the research is that it identifies key tensions that exist, yet it's still not clear how commonplace the various views are. Second, it emerges from limited experience. Most of these tools have been developed in the past few years and are not mainstream tools yet. In some cases, participants 
projected hypothetical uses in the workplace based on their experiences with consumer technologies. Continued research is necessary as professionals gain more hands-on experience with these tools.

Since recorded meetings are increasingly common, we recommend continued research about the ethical use of recorded meeting data. We encourage scholars to study specific applications and goals (e.g., team dynamics reports, hiring and promotion decisions, organizational engagement) of algorithmic tools to recorded meeting data. We also encourage scholars to continue to study the cross-cultural differences in privacy expectations.

\section{Summary}

Meeting recordings and algorithmic tools that process and evaluate recorded meeting data may provide many new opportunities for employees, teams, and organizations. Yet, the use of this data raises important consent, data use, and privacy issues. This research demonstrated key tensions that should be addressed in organizational policymaking: disruption versus help in relationships, privacy versus transparency, employee control versus management control, learning versus evaluation, and trust in AI versus trust in people. We recommend that organizations adopt a social contract approach to setting policy and guidelines for recorded meeting data.

\section{References}

[1] S. Patnaik, "Zoom's daily active users jumped from 10 million to over 200 million in 3 months," Reuters, 2 April 2020. [Online]. Available: https://venturebeat.com/2020/04/02/zooms-dailyactive-users-jumped-from-10-million-to-over-200million-in-3-months/. [Accessed 12 July 2020].

[2] D. Bhave, L. Teo and R. Dalal, "Privacy at work: A review and a research agenda for a contested terrain," Journal of Management, vol. 46, no. 1, pp. 127-164, 2020.

[3] J. Burnett and T. Lisk, "The future of employee engagement: Real-time monitoring and digital tools for engaging a workforce," International Studies of Management \& Organization, vol. 49, pp. 108-119, 2019.

[4] C. Fleischmann, P. Cardon and J. Aritz, "Smart collaboration in global virtual teams: The influence of culture on technology acceptance and communication effectiveness," in Proceedings of the 53rd Hawaii International Conference on System Science, Maui, Hawaii, 2020.

[5] C. Cutter and R. Feintzeig, "Smile! Your boss is tracking your happiness," The Wall Street Journal, p. B7, 7 March 2020.
[6] C. Cutter, T.-P. Chen and S. Krouse, "You're working from home, but your company is still watching you. .," 18 April 2020. [Online]. Available: https://www.wsj.com/articles/youre-working-fromhome-but-your-company-is-still-watching-you11587202201. [Accessed 13 July 2020].

[7] P. Leonardi and N. Contractor, "Better people analytics," Harvard Business Review, pp. 70-81, November-December 2018.

[8] K. Lix, A. Goldberg, S. Srivastava and M. Valentine, "Expressing different: Interpretive diversity and team performance," Standford University, Palo Alto, 2019.

[9] P. Gloor, C. Fronzetti and F. Grippa, "The digital footprint of innovators: Using email to detect the most creative people in your organization," Journal of Business Research, vol. 114, pp. 254-264, 2020.

[10] R. Daft and R. Lengel, "Organizational information requirements, media richness and structural design," Management Science, vol. 32, no. 5, pp. 554-571, 1986.

[11] A. R. Dennis, R. M. Fuller and J. S. Valacich, "Media, tasks, and communication processes: A theory of media synchronicity," MIS Quarterly, vol. 32, no. 3, pp. 575-600., 2008

[12] I. Seeber, L. Waizenegger, S. Seidel, S. Morana, I. Benbasat and P. Lowry, "Collaborating with technology-based autonomous agents: Issues and research opportunities," Internet Research, vol. 30, no. 1, pp. 1-18, 2020.

[13] E. Stone and D. Stone, "Privacy in organizations: Theoretical issues, research findings, and protection mechanisms," in Research in personnel and human resources management, Vol. 8, 411, JAI Press, 1990, p. 349.

[14] E. Stone-Romero and D. Stone, "Current perspectives on privacy in organizations," in Managing social and ethical issues in organizations, Information Age, 2007, pp. 325-362.

[15] E. Stone-Romero, D. Stone and D. Hyatt, "Personnel selection procedures and invasion of privacy," Journal of Social Issues, vol. 59, pp. 343-368, 2003.

[16] A. Acquisti, L. Brandimarte and G. Loewenstein, "Privacy and human behavior in the age of information," Science, vol. 347, pp. 509-514, 2015.

[17] M. Adams, "Big data and individual privacy in the age of the Internet of Things," Technology Innovation Management Review, vol. 7, no. 4, pp. 12-24, 2017.

[18] U. Leicht-Deobald, T. Busch, C. Schank, A. Weibel, S. Schafheitle, I. Wildhaber and G. Kasper, "The challenges of algorithm-based HR decision-making for personal integrity," Journal of Business Ethics, vol. 160, pp. 377-392, 2019.

[19] S. Petronio, Boundaries of privacy: Dialectics of disclosure, Albany: State University of New York Press, 2002.

[20] S. Trepte, L. Reinecke, N. Ellison, O. Quiring, M. Yao and M. Ziegele, "A cross-cultural perspective on the 
privacy calculus," Social Media + Society, vol. 3, no. 1, pp. 1-13, 2017.

[21] Z. Liu and X. Wang, "How to regulate individuals' privacy boundaries on social network sites: A crosscultural comparison," Information \& Management, vol. 55, no. 8, pp. 1005-1023, 2018.

[22] L. Francis and J. Francis, Privacy: What everyone needs to know, Oxford: Oxford University Press, 2017.

[23] S. Miller and J. Weckert, "Privacy, the workplace and the Internet," Journal of Business Ethics, vol. 28, pp. 255-265, 2000.

[24] U. Gal, T. Jensen and M.-K. Stein, "Breaking the vicious cycle of algorithmic management: A virtue ethics approach to people analytics," Information and Organization, vol. 30, pp. 2-15, 2020.

[25] G. Alder, M. Schminke and T. Noel, "The impact of individual ethics on reactions to potentially invasive HR practices," Journal of Business Ethics, vol. 75, pp. 201-204, 2007.

[26] K. Kellogg, M. Valentine and A. Christin, "Algorithms at work: The new contested terrain of control," Academy of Managment Annals, vol. 14, no. 1, pp. 366-410, 2020.

[27] I. Pollach, "A typology of communicative strategies in online privacy policies: Ethics, power and informed consent," Journal of Business Ethics, vol. 62, no. 3, pp. 221-235, 2005.
[28] R. Donaldson and T. Dunfee, "Toward a unified conception of business ethics: Integrative social contracts theory," Academy of Management Review, vol. 19, no. 2, pp. 252-284, 1994.

[29] K. Martin, "Understanding privacy online: Development of a social contract approach to privacy," Journal of Business Ethics, vol. 137, pp. 551-569, 2016.

[30] K. Martin, "Diminished or just just different: A factorial vignette study of privacy as a social contract," Journal of Business Ethics, vol. 111, pp. 519-539, 2012.

[31] O. Robinson, "Sampling in interview-based qualitative research: A theoretical and practical guide.," Qualitative Research in Psychology, vol. 11, no. 1, pp. 25-41, 2014.

[32] B. Marshall and P. Cardon, "Does sample size matter in qualitative IS studies?," Journal of Computer Information Systems, vol. 54, no. 1, pp. 11-22, 2013.

[33] V. Braun and V. Clarke, "Using thematic analysis in psychology," Qualitative Research in Psychology, vol. 3, no. 2, pp. 77-101, 2006.

[34] R. J. House, P. J. Hanges, M. Javidan, P. W. Dorfman and V. Gupta, Culture, leadership, and organizations: The GLOBE study of 62 societies, Sage, 2004. 